\title{
Reconfiguring Architectural Space Using Generative Design and Digital Fabrication: A Project Based Course
}

\author{
Sherif M. Abdelmohsen \\ Ain Shams University, Egypt \\ sherifmorad@eng.asu.edu.eg
}

\begin{abstract}
This paper discusses a course that addresses the integration between generative design and digital fabrication in the context of reconfiguring architectural space. The objective of the course, offered for 3rd year architecture students at the Department of Architecture, Ain Shams University, Egypt, was to design and fabricate interior design elements to be installed within the department lobby. Students worked in digital and physical environments to develop 8 group projects that featured concepts of shape grammars, Lsystems, fractals and cellular automata. The potential of the realized projects is discussed in terms of 3D development of systems, contextual generative design, and pedagogical objectives.
\end{abstract}

Keywords: Contextual generative design; Rule-based systems; Self-organizing systems; Digital fabrication.

\section{Introduction}

Generative systems have been explored in the architectural design process through different procedures and levels of autonomy to generate architectural form. Whether through a completely automated or user-controlled process, the generative design process typically involves algorithm design and derivation, parameter and form adjustment, and best variant selection. Some of the most exploited generative systems include rule-based systems (shape grammars, L-systems, and fractals), self-organizing systems (cellular automata and swarm systems), and computational geometry (voronoi diagrams and $A^{*}$ algorithms) (Terzidis, 2006). One of the advantages, but at the same time challenges, of generative systems has been the digression from pre-existing notions of conventional vocabulary to address realworld architectural contexts with high levels of complexity and limitations (Arida, 2004). The exercise of precluding abstract formalism to enable effective incorporation of context, defined by Alexander (1964) as "anything in the world that makes demands of the form - including designer, client, user, meaning, aesthetics, environment, and function", has been a growing interest in generative design methods. The digital fabrication and experimentation with the emergent produced form adds another layer of complexity into the physical context realization aspect, allowing for an evaluation of the generated form and its inherent contextual and possibly cultural parameters and meanings.

\section{Course Description and Objectives}

This course was developed as an initiative in the "Computer applications" stream of the architectural curriculum at the Department of Architecture, Ain Shams University, Cairo, Egypt. The program for $3^{\text {rd }}$ year architecture students typically involved computer aided modeling and rendering rather than a comprehensive understanding of computational design, as described by Menges (2009). Besides not being reflected in the program of the department and not being perceived as an essential component of the vocabulary of a designer or architect, the equipment necessary to run such a computational design approach were not accessible within the university resources. Within this challenging environment, the pedagogical goal was to establish awareness as to the significance of such an approach, specifically involving generative and algorithmic design and digital fabrication, in terms of its contribution to the design thinking process and as a necessary skill set required from future architects.

The course built on previous studies and projects (Schein, 2002; Ebnöther, 2004; Kilian, 2006; Schindler and Mbiti, 2011). It focused however on the theme of reconfiguring architectural space, consciously putting into consideration factors of context, scale, and anticipated functionality. The course was designed to meet the following objectives: 1 ) to expose students to concepts of generative and algorithmic design, and walk them through the digital chain experience (from design and modeling to digital fabrication and realization); and 2) to communicate the significance of form finding versus form making within a contextual approach to generative design, in an effort to make students understand and appreciate generative design methods away from abstract formalism that does not respond to basic functional, behavioral and contextual requirements in a given setting.

The course started off by introducing concepts of generative systems, algorithmic and parametric design. Students were divided into 8 groups and were asked to research specifically 4 
domains of generative design; shape grammars, L-systems, fractals, and cellular automata, where two groups per system were assigned to research fundamentals and software respectively. The groups then started brainstorming and developing preliminary ideas for their projects. These brainstorming sessions involved studying the context of application of the projects, which is the main department lobby. The lobby typically featured high densities of students going in and out of two adjacent lecture halls with a capacity of 200 students each, in addition to being a main circulation hub leading from the main corridor to the faculty meeting room. Other spaces on the lobby included two WCs (male and female), a small kitchenette serving students and faculty in that floor, and the main building staircase. Problems arising from this high density and the multitude of activities inspired the students in their approaches, as that lobby was their main gathering space but lacking many basic services.

After understanding the context and identifying the main problem, the students were asked to develop digital models, showing the understanding of the systems and the challenge of implementing them in a 3D configuration, and physical prototypes to demonstrate an understanding of detailing, connections, structural stability and contextual compliance. After prototyping and refinement of ideas, detailed computer models were developed for fabrication into full scale physical models. Throughout project development, students were asked to demonstrate how their systems worked, starting from the initial modular unit, how it was propagated through the system, different propagation scenarios, and evaluating them in terms of context compliance. The required submission included the digitally fabricated full scale model, a poster describing the design process, and a video presentation that describes the conceptual approach, intended use and scenarios of application.

In parallel, and as students were not previously exposed to generative design tools, hands-on sessions were held to introduce the students to Grasshopper, Rhino's graphical algorithm editor. The students were free to use any other software, based on their individual research concerning the required capabilities, functionality and project complexity, but introducing Grasshopper and its generative systems plug-ins was used as a guide for general development.

Along the design process for each of the groups, students were given feedback regarding the conceptual clarity of their projects and contextual compliance, the suitability of the conducted digital and physical modeling procedures, level of detailing and complexity, and the added value of the projects in terms of innovation and contribution. Early on physical prototyping was encouraged in order to perceive potential form, proportion, scale, material attributes, geometric relations, connections and assembly from day one. Students were also encouraged to use additional features such as lighting fixtures and LEDs, speakers, and furniture accessories to enhance the user behavior experience alongside their interior design elements.

\section{Results}

Figure 1 shows the eight student projects as displayed in the department lobby.

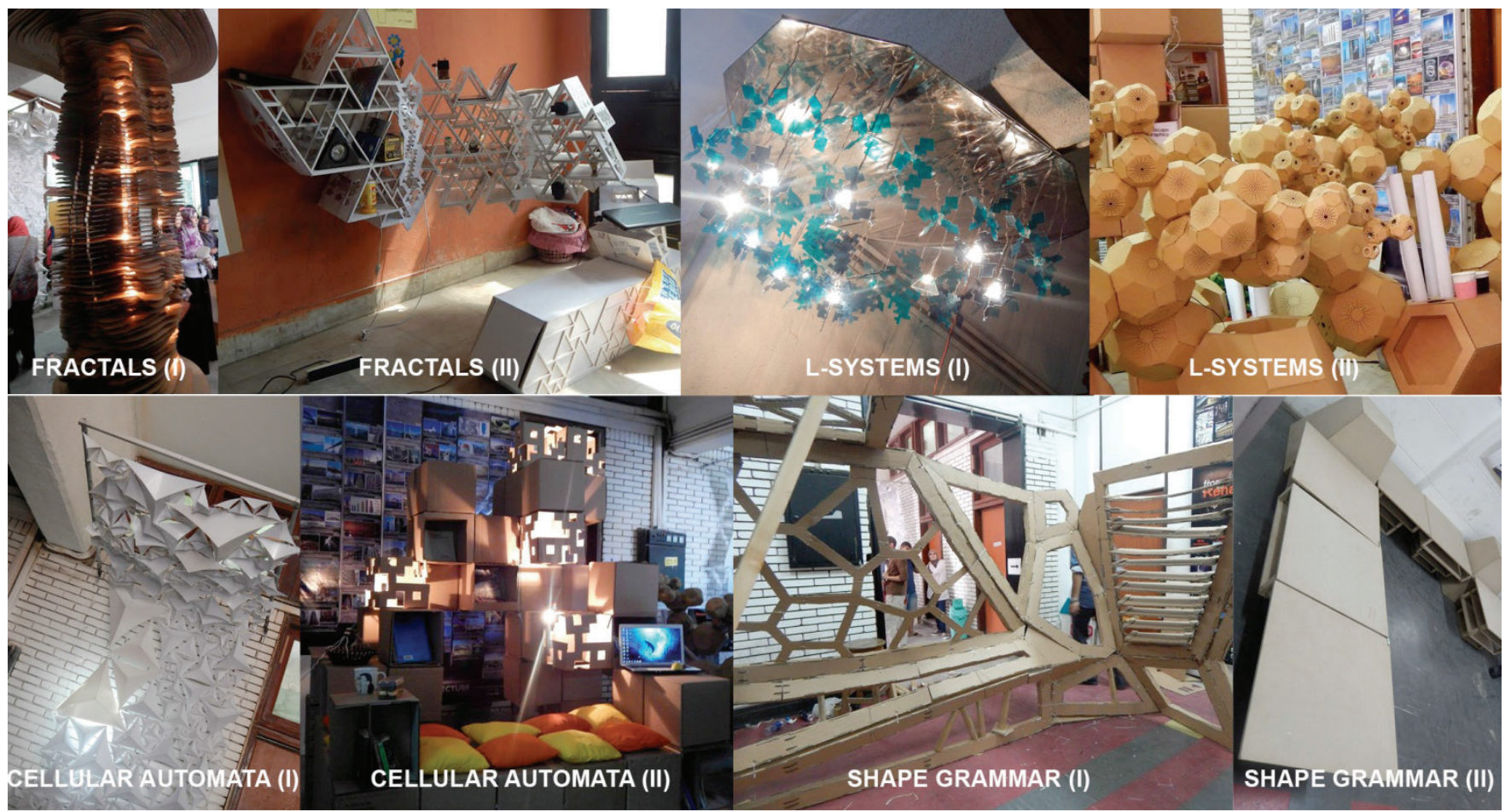

Figure 1: The 8 student group projects, as displayed in the department main lobby 
Four of the eight groups developed a multi-purpose pavilion with seating, lighting and storage features, including a unique experiential and space enclosure setting. One group developed a lighting fixture, one group developed an integrated ceiling-wall unit with lighting units, another developed a column cladding element with storage and shelves, and another developed a reconfigurable seating element. One of the main problems highlighted by the students was the lack of adequate seating areas, services, work surfaces and storage space in the lobby. This was especially unique, as they pointed out the importance of temporary functionality, which was more suitable to the behavior, circulation and passing-by nature of the students in this space. Students usually needed a quick and easy way to place their tools or drawing sheets which varied in size, a handy and accessible surface to put their coffee or belongings, or a temporary work surface to finish up some work on their laptops or discuss their projects with other students or faculty on the fly. At the same time, enclosures with more comfortable seating and focused lighting and light music in the background provided for a more relaxed setting and allowed for social gathering, reading and relaxation. A common observation expressed by the students in their analysis of the context and in their design intent was the need for a different type of social interaction, and the need for change of pace, rhythm of movement and behavior within the lobby space, such that the dynamics of the space become more functionally and socially oriented rather than just being an element of circulation between two points.

In most projects, the implemented generative system informed the design and space reconfiguration process. As the constraint given to the students implied that each of the generative systems was the only means to generate their projects, this allowed for diversity in solution space, affected the design thinking process, and had a clear impact on the nature and features of the resulting designs. This was evident in how the projects were influenced by the inherent characteristics of the used system, for example, the propagation and pattern growth of L-systems, the self similarity of fractals, the state transitions of cellular automata, and the multitude of modifiers and operations in shape grammars.

In terms of software, only two groups (L-systems II and Shape Grammar I) extensively used Grasshopper in their modeling and propagation process. Four other groups started with Grasshopper but decided in the middle of the process to shift to software they were comfortable with, including 3D Max with some plug-ins for parametric generation, or other generative design standalone software. The remaining two groups used CAD software in their process.

This did not come as a surprise due to the time constraints, software challenge and the implied steep learning curve. However, students who explored the full potential of Grasshopper demonstrated thorough, well studied and detailed process, and investigated in depth alternatives and scenarios for unit propagation and form finding. The same group of students had unique ideas that added more value to the contextual adaptation of their projects. The L-systems (II) group focused on the concept of visual access and privacy as a driver for the form of their project (Figure 2). The project was located such that it visually blocks the female WC space from students sitting in the opposite lecture hall or those standing in other parts of the lobby, and the L-system propagation method was directed to suit this goal. The Islamic Mashrabeya was taken as an inspiration for the project form at different levels, including the hierarchy of solid and void percentages between the project modular units and their internal perforations, and the visual privacy component which provides a partial block of view between the public and more private space.

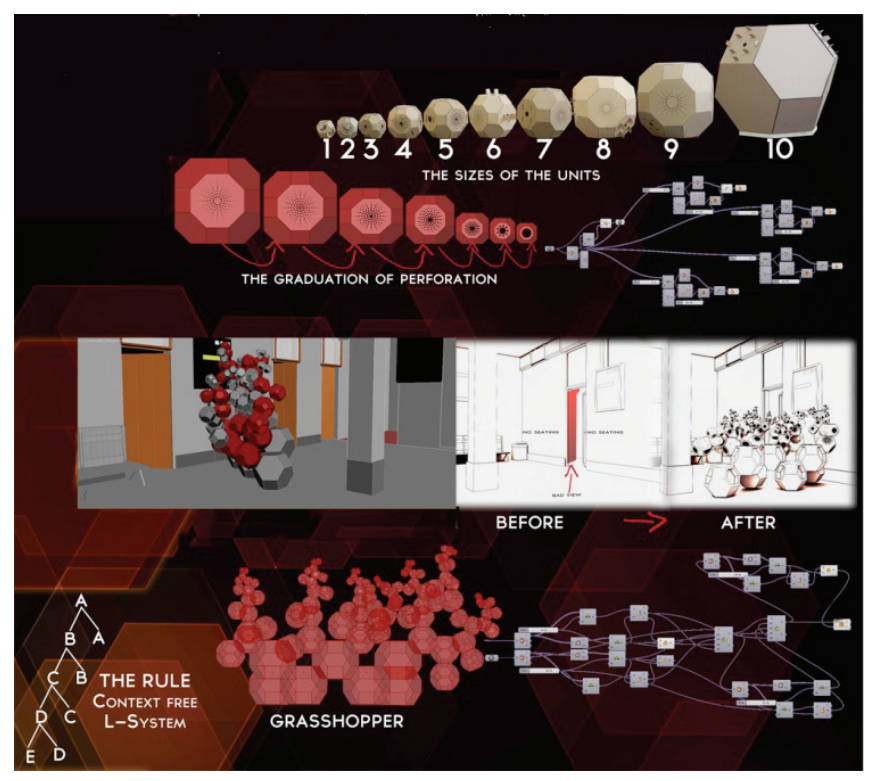

Figure 2: Form finding process for the L-systems (II) group

The Shape Grammar (I) group developed another unique approach which depended on parametrically dividing a given spatial context into desired functional zones and elements of interior design, including seating, surfaces, shelves and storage, based on a substrate algorithm developed in Grasshopper and 3D Voronoi cells, and providing for flexibility of the resulting geometry based on user input and control (Figure 3). The group presented a digital representation of the process, and digitally fabricated one fullscale modular unit. The process defined by the group involves identifying the boundary of the spatial context to be divided, generating partitioned spaces as desired using a substrate algorithm, creating 3D Voronoi cells based on the spatial divisions, manipulating the resulting spatial divisions after assigning functional versus circulation spaces, and using nodes to create façade and flooring patterns. Elements of furniture and interior detailing of the resulting space are carved through the geometry by subtracting the 3D Voronoi cells to generate built-in seating or surfaces. 


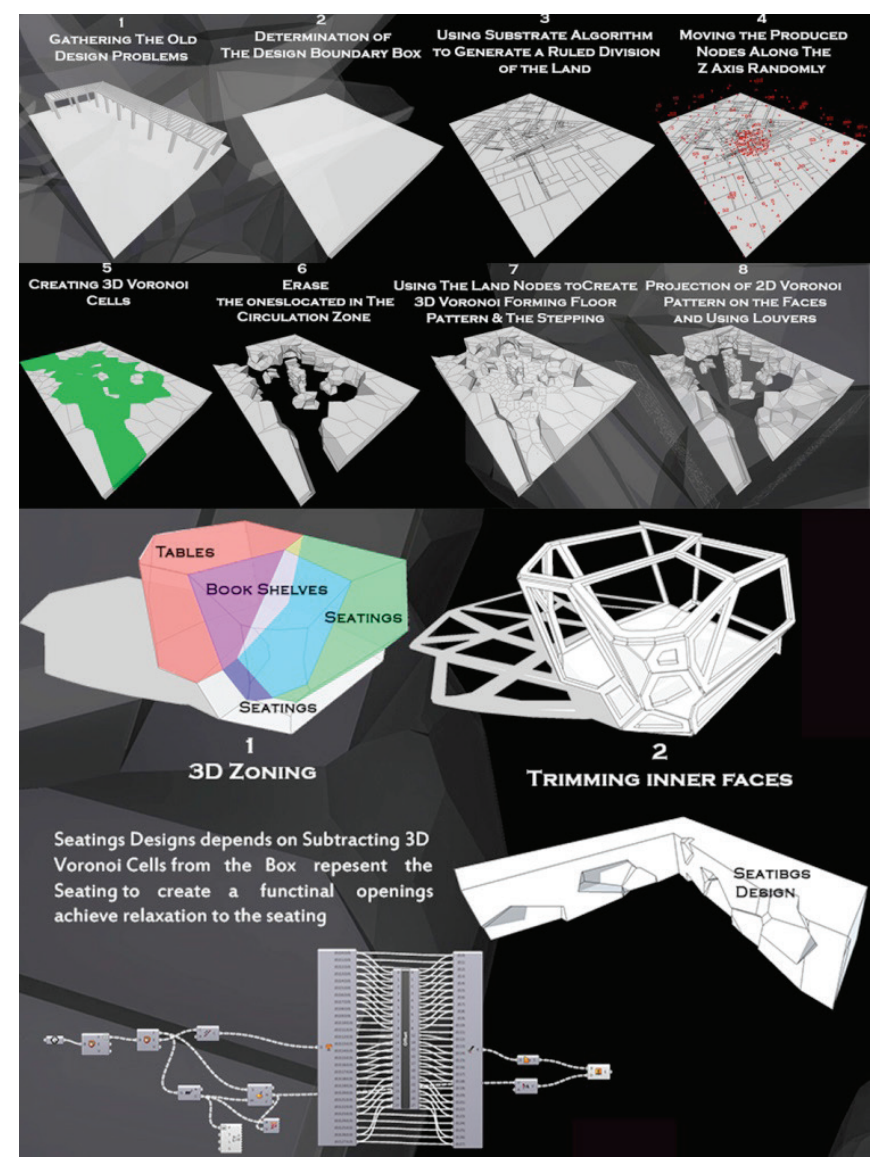

Figure 3: Form finding process for the Shape Grammar (I) group

The cellular automata (II) group developed a multi-functional unit with an attempt to maximize the scenarios for user behavior within the lobby space (Figure 4). The iterations created by the cellular automata self-organizing patterns allowed for studying different cases of use and functionality.

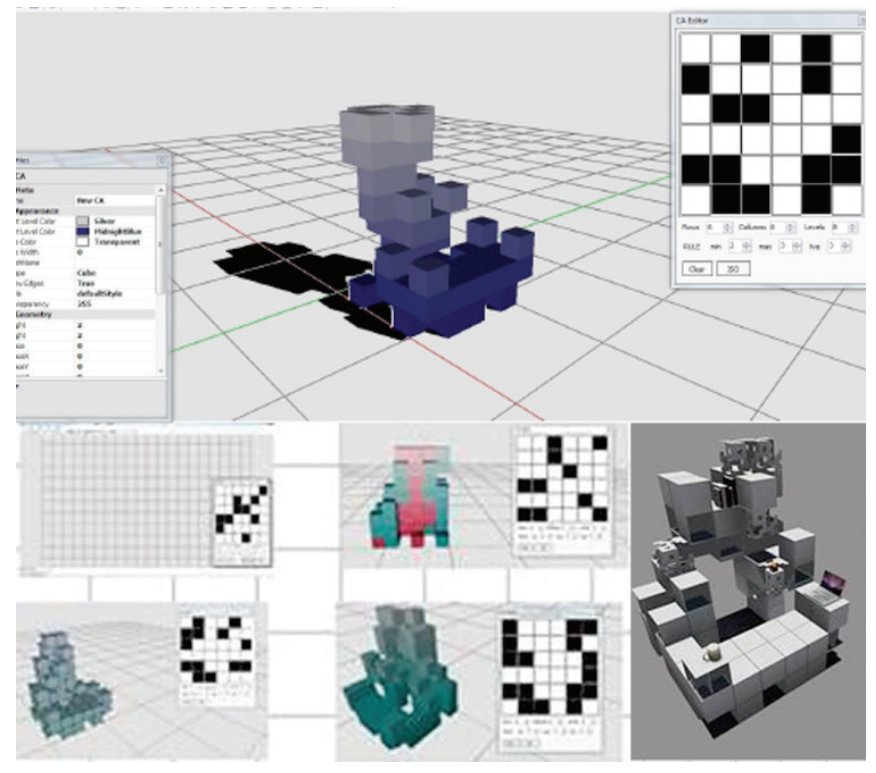

Figure 4: Form finding process for Cellular Automata (II) group
The group used Fun3D, a Visual Basic based software used to explore cellular automata and other $3 D$ and parametric configurations, to study the 3D spatial experience of their unit based on an initial configuration and rule definition. Although the same basic geometric module was used, exploring different scales and multiple solid and void percentages enhanced the spatial experience and allowed for studying elements of light, shading, seating, storage, shelving and circulation.

\section{Conclusions}

The results of the course were generally well appreciated by faculty and students, and the resulting outcome reflected a variety of design approaches and ideas. Issues of concern involved the high cost of fabricated elements, workload, software learning curve, especially Grasshopper, where the task at hand involved the complexity of learning the tool, applying it in 3D to generate form using specific rules, in addition to the complexities of full scale fabrication regarding realization, constructability and detailing.

Regarding the contextual approach which was one of the main goals of this course, almost half of the groups attempted to consciously take the existing setting and its implications into consideration. Other groups could not completely ground their work contextually. It is speculated that expertise with the software played a significant role in justifying the employed contextual approaches. An interesting finding is the variety of these approaches which went far beyond providing just functional needs but extended to include visibility and privacy considerations, accommodating user behavior, circulation and comfort, incorporating historical and cultural references, and engaging users in customizing their spaces through parametric controls. Accessories such as speakers, LEDs, and elements of furniture also added another dimension to context compliance. One of the groups went further to propose an additional responsive alternative that involves adapting dynamic control of specific louvers in their project according to changing environmental conditions and user behavior. Students were advised to carry on their experimentation with their projects beyond the scope of the course, but with more focus on performative evaluation in order to assess their compliance to issues such as structure, context and wayfinding.

An important challenge in this course was realizing the employed generative systems in 3D. Most precedent projects featured 2D applications of these systems as patterns, artwork or 2D geometry rather than $3 \mathrm{D}$ configurations. Most of the available software and plug-ins also do not contain ready-made definitions or algorithmic procedures for 3D propagation or growth patterns. More experienced students overcame this challenge either by developing translations between different software, or by moving back and forth between digital modeling in Grasshopper for example and physical prototyping of the generated 3D units. The issue of pushing for a form finding process rather than a form making process was also challenging. Not all groups could achieve 
a successful form finding process. This was mainly due to spending a long time struggling with software or shifting to a different process and tool, which focused their attention then on arriving at a product rather than an elaborate form generation process.

Students varied in their approach to digitally fabricating and physically realizing their projects. The three groups highlighted in figures 2, 3 and 4 worked extensively throughout the project on developing physical prototypes at different levels of detail and experimenting with several iterations and techniques. The Lsystems group especially went further to explore the effect of different materials and textures, efficient ways of fabrication, and studying multiple methods of connection and detailing. This was reflected in the final product, where those three groups demonstrated a higher level of attention to detail and constructability. Time however was a constraint, especially when groups moved from the prototyping phase to full scale production, where problems began to surface concerning structure, joints and durability. Students were advised to plan for adequate time and task management to make room for unforeseen conditions.

There were many challenges in this course. Most of these challenges were already expected due to various reasons, including the large number of students, scarce resources (especially digital fabrication and rapid prototyping machines) and limited instruction time, being one of the first public universities in Egypt to implement such an approach. However, lessons learned from this experience are numerous. A reduced scale of implementation, or an accumulation of mini-projects and exercises with different educational goals, with more focus on process and performance-based evaluation, could have produced a better learning outcome. Restructuring the curriculum to augment the computer aided design stream with adequate prerequisites would have definitely enhanced the performance and informed the design thinking process.

\section{Acknowledgments}

The author would like to thank all the students (class year of 2014) at Ain Shams University for their efforts in making this course a success, as well as Hazem Eldaly and Ahmed Eid for their continuous technical advice and support. Thanks also due to Mohammad Mekawy, Hisham Bahaa, Mohammad Abdelaziz and Fatma Fathy for assisting with the students' design process. The author would like to express gratitude to Professors Amr Abdelkawi and Yasser Mansour for their valuable feedback and insight as guest critics.

\section{References}

Alexander, C. (1964). Notes on the Synthesis of Form. Cambridge, MA: Harvard University Press.

Arida, S. (2004). Contextualizing Generative Design, MSc Thesis, Department of Architecture, MIT.

Ebnöther, Y. (2004) SkinChair. Diplomarbeit NDS Architektur, ETH Zürich.

Kilian, A. (2006). Design Exploration with Circular Dependencies: A chair design experiment. Proceedings of the CAADRIA 2006 Conference, Kumamoto, Japan, 217-226.

Menges, A. (2009). Uncomplicated Complexity: Integration of Material, Form, Structure and Performance in Computational Design, in Hirschberg, U, Gethmann, D, Böck, I and Kloft, H (eds), GAM.06 Nonstandard structures, Springer Verlag, Wien, 180-193.

Schein, M. (2002). Applied Generative Procedures in Furniture Design, Proceedings of the Generative Art International Conference 2002, Milan, Italy, 21.1-21.10.

Schindler, C. and Mbiti, K. (2011). Urban Furniture: Introducing Parametric Modelling and Digital Fabrication in a Part-time Study, Proceedings of the 29th eCAADe Conference, Ljubljana, Slovenia, 368-373.

Terzidis, K. (2006). Algorithmic Architecture, Routledge. 